\title{
PROFILE OF ANTIBODY RESPONSES, IN CHILDREN WITH CANCER, TREATED WITH SELENIUM
}

K.C. Rocha, J. Carton, T. Gascon, F. Schindler, A.P. Moreira, L. Piton, A. Gonçalves, R.L. Beltrame, F.L.A. Fonseca

Faculdade de Medicina do ABC, Santo André, Brazil

There are reports of globulin abnormalities in various types of tumors during chemotherapy. Recently, even $\mathrm{IgE}$ levels seem to play an important role in the tumor's immune response. Trace elements, like Selenium (Se), act as basic components of essential system which play roles in immunology. Chemotherapy has been associated with decrease concentration of Se and this fact has been correlated with immunodepression states.

Objective: Was to investigate, in children under treatment at the pediatric oncology ambulatory of Faculdade de Medicina do ABC, the immunoglobulin and PCR levels, before and after Se or Placebo (P) supplementation.

Method: A double-blind cross-over study that evaluated 36 patients. IgM, IgG and IgA levels were measured by immunodiffusion. IgE was quantified by chemiluminescence, PCR was determined by an ultrasensible technique.

Results: Leukemia (L) patients (age 7,29 \pm 4 ) presented the following levels before Se or P supplementation: IgM and IgA decreased, IgG, IgE and PCR increased. Solid Tumor (ST) patients (age 8,26 \pm 4 ) presented before supplementation, increased levels of IgM, IgG, IgA, IgE AND PCR. IgA and IgE (ST) increased more than $\mathrm{L}$ patients before supplementation $(\mathrm{p}<0,05)$. IgE and $\operatorname{IgG}(\mathrm{L})$ decreased in most of patients during $\mathrm{Se}$ supplementation.

\section{Conclusions:}

1. Se supplementation didn't alter immunoglobulin production in ST;

2. IgA and IgM in L was not modified by Se;

3. Most of L patients experienced decreased levels of IgE and IgG during Se supplementation;

4. The majority of patients (L or ST) increased levels of IgE and IgG.

5. The studied patients did not present hypogammaglobulinemia. 